\title{
Postcolonial : des mythes à l'enseignement de l'histoire du fait colonial
}

NOTE CRITIQUE SUR Étienne ACHILLE \& Lydie MOUDILENO (2018). Mythologies postcoloniales. Pour une décolonisation du quotidien. Paris, Honoré Champion ; Et Laurence DE COCK (2018). Sur l'enseignement de l'histoire. Débats, programmes et pratiques de la fin du XIX ${ }^{\mathrm{e}}$ siècle à nos jours. Montreuil, Libertalia.

Postcolonial: from myths to teaching the history of the colonial fact

\section{Carole Reynaud-Paligot}

\section{(2) OpenEdition}

Édition électronique

URL : https://journals.openedition.org/bssg/754

DOI : $10.4000 /$ bssg.754

ISSN : 2490-9424

Éditeur

Université Paris Lumières

Référence électronique

Carole Reynaud-Paligot, « Postcolonial : des mythes à l'enseignement de I'histoire du fait colonial », Biens Symboliques / Symbolic Goods [En ligne], 9 | 2021, mis en ligne le 27 décembre 2021, consulté le 13 janvier 2022. URL : http://journals.openedition.org/bssg/754 ; DOI : https://doi.org/10.4000/bssg. 754

Ce document a été généré automatiquement le 13 janvier 2022.

Biens Symboliques / Symbolic Goods 


\section{Postcolonial : des mythes à l'enseignement de l'histoire du fait colonial}

NOTE CRITIQUE SUR Étienne ACHILLE \& Lydie MOUDILENO (2018). Mythologies postcoloniales. Pour une décolonisation du quotidien. Paris, Honoré Champion ; Et Laurence DE COCK (2018). Sur l'enseignement de l'histoire. Débats, programmes et pratiques de la fin du XIX ${ }^{\mathrm{e}}$ siècle à nos jours. Montreuil, Libertalia.

Postcolonial: from myths to teaching the history of the colonial fact

\section{Carole Reynaud-Paligot}

\section{RÉFÉRENCE}

Étienne ACHILLE \& Lydie MOUdiLENo. Mythologies postcoloniales. Pour une décolonisation du quotidien. Paris, Honoré Champion Laurence DE COCK. Sur l'enseignement de l'histoire. Débats, programmes et pratiques de la fin du XIX ${ }^{e}$ siècle à nos jours. Montreuil, Libertalia 
Etienne Achille et Lydie Moudileno

\section{Mythologies postcoloniales}

Pour une décolonisation du quotidien

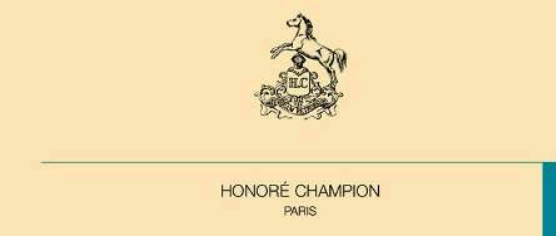

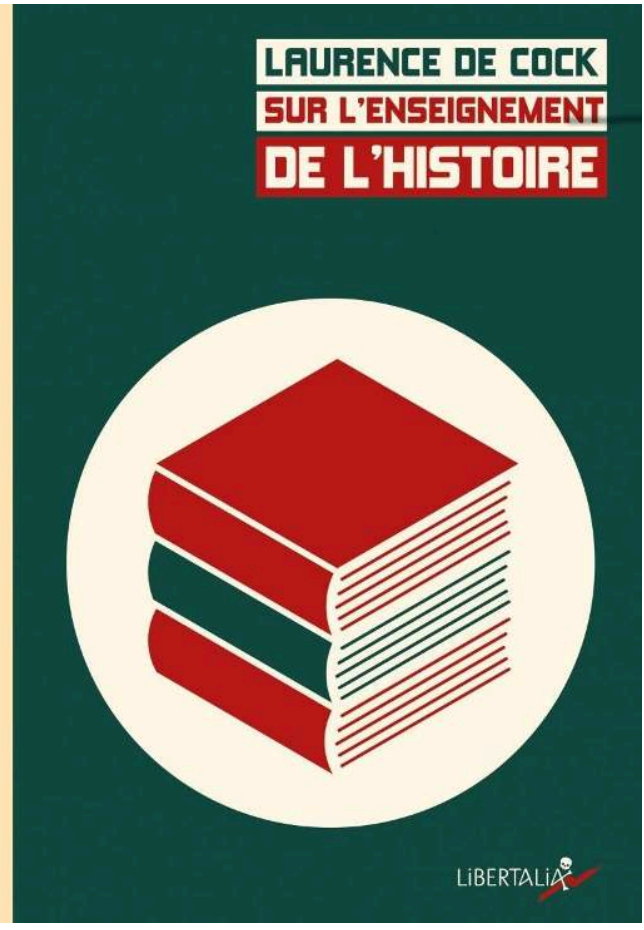

LRURENCE DE COCK SUR L'ENSEITNEMENT DE L'HITTOIRE

De gauche à droite: première de couverture de l'ouvrage de Étienne ACHILLE \& Lydie moudiLENo (2018), Mythologies postcoloniales. Paris, Honoré Champion; Etle première de couverture de l'ouvrage de Laurence dE соск (2018), Sur l'enseignement de l'histoire. Montreuil, Libertalia.

(C) Honoré Champion (C) Libertalia

1 Les ouvrages présentés dans le titre représentent deux études sur la question du "postcolonial». Étayés scientifiquement, ces deux ouvrages apportent un éclairage intéressant sur cette thématique. Le premier montre que la volonté d'inscrire l'enseignement colonial dans les programmes scolaires a été présente dès les années 1980 : la volonté est alors indéniable mais la réalisation de l'objectif a été, et est toujours, confrontée à des obstacles idéologiques. La seconde enquête, qui n'a pas la même ampleur, invite plutôt à une réflexion sur une emprise coloniale présente jusque dans le quotidien, tout en illustrant cette approche par quelques études de cas.

Laurence de Cock entraîne son lecteur dans la fabrique des programmes scolaires, et plus spécifiquement dans les soubresauts provoqués par l'intégration de l'enseignement du passé colonial dans ces programmes. Pris entre des enjeux politiques et idéologiques, l'enseignement $\mathrm{du}$ fait colonial doit faire face aux nombreux enjeux auxquels la société française est confrontée depuis les années 1980 : le "problème de l'immigration », les thématiques et revendications identitaires, les enjeux mémoriels.

De la fin du $\mathrm{XIX}^{\mathrm{e}}$ siècle jusqu'à la décolonisation, l'enseignement colonial s'est parfaitement intégré aux valeurs républicaines et au «roman national ». La « mission civilisatrice " fait alors pleinement partie de l'identité républicaine. Des générations d'écoliers ont intégré cette mission tout comme les représentations inégalitaires et hiérarchisantes qui les accompagnaient. La remise en cause de ces éléments de l'identité républicaine a entraîné une crise de l'enseignement de l'histoire: l'enseignement d'une analyse critique du rôle de la France dans le processus de colonisation s'est depuis les années 1970 heurté à des enjeux idéologiques et a provoqué débats, polémiques et affrontements. L'enseignement du fait colonial a été 
soumis à une double pression, venant d'une part de ceux qui déplorent la censure et les tabous conduisant à un sous-enseignement, d'autre part de ceux qui déplorent l'idéologie de la « repentance ».

4 L'enquête très sérieuse que conduit Laurence de Cock en révèle les différents épisodes grâce à l'exploration de fonds d'archives (Conseil national des programmes, bureau de programmes d'enseignement, Direction générale de l'enseignement scolaire, Fonds des cabinets ministériels, etc.) mais aussi d'observations participantes.

5 L'historienne situe le premier moment de cette histoire dans les années 1970, dans un contexte marqué par les premières commémorations médiatiques, par l'organisation politique des jeunes immigrés mais aussi par la construction du «problème de l'immigration ». L'ethnicisation de la question migratoire conduit à une reformulation sous l'angle de l'origine: les travailleurs immigrés deviennent les "immigrés maghrébins ». Du côté des militants et chercheurs qui s'inquiètent de la montée du racisme dans la société française, l'approche «interculturelle» prévaut, les savoirs universitaires prônent les "relations interculturelles » : la connaissance entre cultures et entre peuples doit conduire à des relations harmonieuses. C'est le temps de la valorisation des cultures qui passe par la mise en avant du folklore, de la musique et de la cuisine. Cette approche se traduit dans l'enseignement par une ouverture aux civilisations "extra-occidentales » sous l'égide de l'UNESCO. Les programmes dits «Braudel», qui défendent une approche anthropologique dénuée de toute historicité, en sont l'emblème (De Cock 2018 : 45). Cependant, contournés par les enseignants, ils sont abandonnés rapidement au seuil des années 1980. La réaction à cette ouverture ne se fait, en effet, pas attendre: les nostalgiques d'une histoire nationale s'insurgent contre sa disparition et orchestrent une campagne médiatique. La controverse bat son plein au début des années 1980, prenant parfois des accents barrésiens lorsque Michel Debré, acteur de premier plan, affirme que «les phénomènes de massification précipitent le déracinement des individus » (De Cock 2018 : 55).

Dès son arrivée au pouvoir, la gauche engage une réflexion sur l'enseignement de l'histoire. Le ministre de l'Éducation Alain Savary mandate l'historien René Girault pour lancer une vaste consultation d'universitaires, d'enseignants, de syndicats, d'associations. Découverte des autres, respect, tolérance sont au programme sans que l'enseignement du passé colonial soit encore central. La question de la scolarisation des enfants d'immigrés est alors l'une des priorités du ministère de l'Éducation. Le ministre Jean-Pierre Chevènement, qui succède à Alain Savary en 1984, missionne l'historien Jacques Berque. Le groupe de réflexion mis en place intègre une vingtaine de personnalités, dont le sociologue Abdelmalek Sayad qui ne tarde pas toutefois à le quitter. Les archives privées de ce dernier font état de son inquiétude: face à la catégorisation et à l'essentialisation culturelle, le sociologue prône la non dissociation des problématiques migratoire et sociale. Les différentes versions du rapport montrent, en effet, les tensions entre la volonté de reconnaître les cultures et le risque de les essentialiser et d'accentuer le différentialisme. Le rapport final semble bien clore les beaux jours de l'interculturalité: il s'agit maintenant d'épargner aux enfants d'immigrés toute forme d'enfermement dans des spécificités culturelles, de "défolkloriser » les contenus et les pratiques pédagogiques. Berque alerte sur les risques de paternalisme, mais aussi de réduction des cultures à des "âges d'or». Ce rapport restera lettre morte. 
7 Les années 1980 sont également marquées par l'irruption de la guerre d'Algérie au sein de l'espace public et des programmes scolaires dans le contexte des premiers succès électoraux du Front national. L'histoire du temps présent trouve sa place au sein des milieux universitaires tout comme au sein des programmes scolaires. Les nouveaux programmes de terminale de 1982, qui remplacent ceux de 1959, s'étendent de la Seconde Guerre mondiale à nos jours : rencontrant un grand écho médiatique, ils doivent faire très vite face aux doléances associatives et parlementaires. La colère des anciens combattants de la guerre d'Algérie qui protestent contre la « remise en cause de la conduite des soldats français pendant la guerre d'Algérie » (De Cock 2018: 76) est visible dans les courriers et pétitions qui s'accumulent. Ces résistances n'empêchent pas pour autant l'installation de la guerre d'Algérie dans les enseignements, dans les sujets d'épreuves des lycées tout comme dans ceux des concours d'enseignants.

8 Enfin, les années 1970-1980 sont également le temps de l'éducation aux droits de l'homme, une éducation impulsée dès les années 1970 par l'UNESCO. Tous les États membres sont encouragés à développer cette thématique, du préscolaire à l'université, mais aussi à être vigilants sur les contenus des programmes scolaires, contenus susceptibles de nourrir la méfiance et le racisme. L'éducation aux droits de l'homme se prolonge par une éducation au développement qui prône une solidarité avec les pays $\mathrm{du}$ " tiers-monde » : ces deux thématiques sont intégrées aux programmes scolaires du début des années 1980 mais soulèvent les critiques de l'APHG, l'association des professeurs d'histoire-géographie.

9 Les années 1990 transforment la guerre d'Algérie en un véritable problème public, tandis que la "construction du problème musulman " se traduit par une focalisation sur l'Islam et par l'émergence d'une «communauté musulmane» qui cristallise l'attention et les inquiétudes avant d'être perçue comme la source d'un « communautarisme» (De Cock 2018: 96). L'année 1989 voit une médiatisation sans précédent du refus de jeunes musulmanes de céder à l'injonction du principal de leur collège d'ôter leurs foulards. Dans ce contexte à fortes polémiques, la connaissance et donc l'enseignement du fait colonial apparaissent à certains acteurs comme un moyen de pacifier les tensions. La guerre d'Algérie devient un objet mémoriel. Pendant la guerre, les premiers travaux d'historiens avaient déjà été publiés, et, à leur suite, plusieurs générations d'historiens avaient poursuivi des recherches. Les années 1990, néanmoins, sont celles qui connaissent la plus forte inflation de travaux, alors que les médias se focalisent sur la jeunesse des quartiers populaires. Là encore, l'insuffisance de l'enseignement du fait colonial resurgit dans le débat public : cette histoire peu ou mal enseignée, refoulée, alimenterait la révolte des jeunes. Ces diagnostics ne sont pourtant guère vérifiés, programmes et manuels insèrent bel et bien l'enseignement de la guerre d'Algérie dans les chapitres portant sur la décolonisation. Les polémiques continuent pourtant, alimentées par ceux qui considèrent que cet enseignement est trop lacunaire ou partisan. Enquêtes, colloques, universités d'été soutenus ou organisés par les pouvoirs publics se succèdent alors que ces derniers entreprennent des commémorations d'évènements comme celui du massacre du 17 octobre 1961.

10 Pour la première fois, en 1990, une administration spécifique est chargée de l'élaboration des programmes. Le Conseil national des programmes (CNP) créé par Lionel Jospin, alors ministre de l'Éducation Nationale, rassemble 22 membres nommés par le ministre: des universitaires, des enseignants, des membres d'associations. Lors de l'installation du CNP, Jospin rappelle la dimension civique de l'enseignement de 
l'histoire, vecteur de l'apprentissage de la tolérance, du respect des « différences » mais aussi sa contribution à la "mémoire collective ". L'acquisition de cette mémoire lui apparaît «d'autant plus indispensable [...] dans des sociétés industrielles et urbaines génératrices d'espaces sans racines ni profondeurs" (De Cock 2018 : 149). Des groupes de travail disciplinaires sont instaurés. Un groupe d'experts sous la houlette de l'historien Jean-Clément Martin est chargé de l'écriture des programmes d'histoire, non sans provoquer des rancœurs auprès des acteurs traditionnels, comme l'association des professeurs d'histoire-géographie (APHG), qui se sentent écartés. Le nouveau programme, qui laisse une large place au fait colonial, en abordant la question des " colonisations et impérialismes ", puis celle des « décolonisations » en intégrant aussi la guerre d'Algérie, suscite des oppositions. L'historien Pierre Chaunu s'insurge contre l'invasion de l'histoire trop contemporaine. Le groupe histoire-géographie de l'IGEN (Inspection générale de l'Éducation nationale) refuse la notion d'impérialisme, et il réclame le maintien, allégés, des programmes en vigueur depuis 1988. Face à ces résistances, les nouveaux programmes sont abandonnés et J.-C. Martin démissionne. En 1993, Serge Berstein lui succède, tandis que Luc Ferry prend la tête du CNP. De nouveaux programmes sont adoptés : en 1995 ceux du collège puis en 1999 ceux du lycée. L'étude de l'émancipation des peuples dépendants est présentée comme distincte de l'histoire nationale, seule à même de procurer aux jeunes Français les éléments d'une mémoire nationale. Les ministères changent; les groupes de travail deviennent des groupes d'experts. Un projet de manuel unique achoppe.

11 Alors que les programmes de 1959 étaient restés en vigueur pendant plus de vingt ans, à partir des années 1980, chaque décennie connaît une, voire deux réformes : nouveaux programmes des collèges en 2008 puis en 2016, nouveaux programmes des lycées en 2010 et en 2018. Le CNP disparait. Le contexte mémoriel est marqué par l'adoption de la loi du 10 mai 2001 sur la commémoration de l'abolition de l'esclavage, loi qui est le fruit d'une mobilisation associative et de la détermination de la députée de Guyane, Christine Taubira. La loi entend apaiser les «mémoires blessées ", restaurer l'estime de soi de ceux dont « la conscience et l'imaginaire portent encore les stigmates de cette abomination" mais aussi lutter contre le racisme en le reliant à ses "sources historiques ». La loi demande aux manuels et aux programmes de recherche en histoire et sciences humaines d'accorder «à la traite négrière et à l'esclavage la place conséquente qu'ils méritent». Cette initiative provoque des débats sur le rôle du Parlement dans l'écriture de l'histoire scolaire. C'est à la même époque qu'est entreprise l'adaptation des programmes dans les départements d'outre-mer. L'universalisme reste la règle puisque les programmes demeurent identiques à ceux de la métropole: seuls les horaires d'enseignement de l'esclavage, des traites et des empires coloniaux sont augmentés. En 2004 est mis en place le Comité pour la mémoire et l'histoire de l'esclavage (CPMHE).

Les réformes des programmes du collège en 2008 puis en 2016 sont à nouveau très marquées par des enjeux idéologiques. En 2008, le nouveau programme du collège qui fait une large place à l'enseignement de l'histoire de la colonisation, de la traite et de l'esclavage, au racisme colonial, suscite à nouveau une mobilisation fortement médiatisée de l'autre camp qui fustige la « repentance » et la disparition de Napoléon. En 2016, ils demandent le rétablissement du «roman national » et déplorent que le "pluralisme culturel favorise l'étude de l'islam au détriment de l'héritage chrétien de la nation française » (De Cock 2018 : 270). Cependant, là encore, on peut constater que le « roman national » n'est pas l'apanage des pensées conservatrices, comme l'illustre la 
déclaration, en mai 2015, de François Hollande, qui affirme que l'enseignement de l'histoire « doit rappeler les heures glorieuses de notre passé ». C'est aussi le retour à une histoire qui se veut nationale et un abandon d'une histoire globale ou connectée, ainsi qu'une moindre importance accordée à l'esclavage et à la colonisation. Cette première mouture s'infléchit sous la pression du CPMHE et du collectif Aggiornamento qui dénoncent son caractère national et européanocentré.

très documentée des débats montre les interférences de ceux-ci dans la fabrique des programmes mais aussi dans les controverses scientifiques sur des notions comme celle de " continuité coloniale ", notion qui répond à des enjeux identitaires et médiatiques et que certaines contributions scientifiques remettent en cause. La notion de « diversité » fait quant à elle l'objet d'une « neutralisation politique » (De Cock 2018 : 206). Le CNP, qui avait été supprimé en 2005 , est remplacé par le Conseil supérieur des programmes créé par François Hollande, dont la composition diffère sensiblement puisqu'il intègre trois députés et trois sénateurs, deux membres du Conseil économique et social et qu'il réduit la place des enseignants et des universitaires.

Ce livre, qui s'appuie sur la consultation d'archives, montre que l'enseignement du fait colonial demeure soumis à de très forts enjeux idéologiques et politiques. À côté de ces enjeux, il faut ajouter les rivalités institutionnelles mais aussi les contraintes d'ordre plus technique liées à la fabrication de manuels, c'est-à-dire les contraintes d'horaires, de pagination, de lisibilité, autant de logiques qui président aussi aux arbitrages finaux. Les renouvellements historiographiques sont sinon les grands absents de cette histoire, $\mathrm{du}$ moins des acteurs de second plan. Ils ne semblent parvenir à infléchir les programmes que lorsqu'ils s'insèrent dans des logiques mémorielles et captent l'attention des médias.

15 L'historienne souligne que l'enquête est à poursuivre afin de cerner ce qu'il reste de ces programmes dans la mémoire des élèves. Il faudrait ajouter, à mon sens, des enquêtes sur les pratiques des enseignants : comment s'approprient-ils ces programmes ? Quelles sont les résistances idéologiques, les contraintes matérielles qui entravent leur mise en œuvre? Les productions audio-visuelles tiennent aujourd'hui une grande place dans les classes, parfois sans être accompagnées d'une démarche pédagogique, alors que le manque de temps est l'argument suprême qui justifie le sacrifice des mondes extraoccidentaux.

16 Loin de céder au raccourci qui consiste à dénoncer la permanence du colonialisme dans la société actuelle sans le démontrer, Étienne Achille et Lydie Moudileno mettent au jour quelques-unes des traces de la mémoire coloniale et des permanences de stéréotypes coloniaux dans la culture populaire d'aujourd'hui. Ils invitent à décoloniser le quotidien, un quotidien qui est devenu, depuis les travaux d'Henri Lefebvre, Roland Barthes, Michel de Certeau ou encore Georges Perec, un terrain digne d'intérêt pour l'étude des représentations et des constructions des identités. La race, comme catégorie imaginaire, continue de sévir au sein de celui-ci. Il en est de même des stéréotypes et du racisme. En suivant les pas de Barthes, les auteurs invitent à traquer non plus les mythes qui servent l'idéologie bourgeoise mais ceux qui portent encore l'idéologie coloniale. Se défendant d'effectuer des parallèles faciles entre la culture coloniale d'hier et la société d'aujourd'hui, les auteurs entendent mettre au jour les traces de l'imaginaire colonial encore présentes dans notre vie quotidienne. Les plaques de rue nous rappellent toujours les héros de l'épopée coloniale : "alors que la dernière rue Pétain a été débaptisée en mars 2013 (Belrain, Meuse), que faire de ces rues, écoles, 
places Bugeaud et autres toponymes des héros militaires de la IIIe République » (32) s'interrogent les auteurs. Ce débat a trouvé très récemment une résonance particulière en France à l'initiative de manifestants et d'associations qui appellent à débaptiser ces rues. Cet examen de la toponymie des rues françaises participerait ainsi à la « démystification de la mémoire républicaine » (34).

Les émissions populaires de la télévision française mobilisent les représentations d'une France rurale au sein de laquelle l'immigration d'origine coloniale serait absente de notre histoire, tandis que les journaux télévisés véhiculent des stéréotypes : «le mythe de La Plus Petite France sert ainsi le mythe de deux France: l'une peuplée de vrais Français solidaires et acquis à la cause d'une identité nationale à préserver et d'où le postcolonial est évacué pour être confiné dans l'autre France, celle ayant perdu sa francité, envahie par des barbares et métèques, vouée à l'incivilité et au désordre » (44). Une humoriste dérape, puis s'excuse, après voir imité la Garde des Sceaux, Christiane Taubira, avec un accent douteux, un sketch qui nous rappelle ceux de Michel Leeb, qui, il n'y a pas si longtemps, dans les années 1980, pouvaient faire rire en mobilisant des stéréotypes coloniaux sur les Africains. Certains chocolatiers continuent à réaliser des chocolats empreints, eux aussi, de stéréotypes coloniaux, en proposant dans leur vitrine des «Bamboula» et des «Negro». Le cinéma populaire s'illustre dans les " comédies multiculturelles» qui prétendent refléter les angoisses identitaires d'une "vraie France ». De telles études sont à poursuivre car elles ne se contentent pas d'asséner des injonctions générales à cesser d'ignorer le postcolonial de notre société mais entreprennent de dévoiler la présence de stéréotypes coloniaux dans notre quotidien. Tout en soulignant l'apparition d'autres représentations et une prise de conscience depuis une quinzaine d'années, les auteurs concluent: «[...] il reste beaucoup à faire. Notre travail en appelle donc d'autres... » (130)

Au-delà de cette mise au jour indispensable des stéréotypes coloniaux, il semble nécessaire d'interroger les raisons de ces permanences. Les générations qui ont été imprégnées de culture coloniale disparaissent, les manuels scolaires ne présentent plus l'inégalité des races mais tentent de promouvoir l'anti-racisme. C'est ce paradoxe que ces deux ouvrages contribuent à éclairer.

\section{AUTEURS}

\section{CAROLE REYNAUD-PALIGOT}

Université de bourgogne/Université Panthéon-Sorbonne/Centre d'histoire du XIX siècle 